\section{FACSORT: A program promoting efficient interpretation of factor analysis output}

\author{
RUSS V. C. REYNOLDS \\ Ohio University, Athens, Ohio \\ and Medical University of South Carolina \\ Charleston, South Carolina \\ and

\section{DAVID L. TOBIN and EDWARD S. LOH Ohio University, Athens, Ohio}

The use of multivariate analysis, especially factor analysis, has grown considerably with the advent of high-speed mainframe computers. However, factor analysis interpretation remains a tedious task. For example, exploratory factor analysis often requires the study of several factor structure matrices derived from the same data set.

FACSORT, a program for sorting factor loadings, relieves investigators of the drudgery and human error associated with manually sorting factor analysis output. Although statistical packages perform some of the operations performed by FACSORT, none offers the complete set of interpretive functions provided by FACSORT.

FACSORT is an interactive set of programs, written for use under the IBM VM/SP (CMS), designed to sort matrices of factor loadings produced by SPSS, SPSSX, and SAS factor analysis procedures. Because FACSORT is designed as an interactive program, it requires that the user have some familiarity with basic CMS operations. Current FACSORT capabilities allow sorting of factor loading matrices with up to 10 factors and up to 300 variables (e.g., questionnaire items). Sorting a matrix of greater than 10 factors is possible with minor manipulation of the source factor structure matrix.

Program Input. FACSORT requires that a factor loading matrix and variable description file be available in the virtual environment. As stated above, FACSORT is designed to operate with factor loading matrices produced by SPSS, SPSSX, and SAS factor analysis procedures; however, any correlation or loading matrix may be used with FACSORT if it contains variables or items that share a common root, as identified in the first column of the matrix (e.g., test ${ }_{1}$, test ${ }_{2}, \ldots$ test $_{n}$ ). Other necessary FAC-

R. V. C. Reynolds's address is: Department of Psychology, Box 85, Porter Hall, Ohio University, Athens, OH 45701.
SORT files include a variable description file, which must contain one-line descriptions (maximum 80 characters) of each variable included in the factor loading matrix (e.g., questionnaire item descriptions).

The remaining FACSORT input consists of sorting parameters, such as the number of factors and variables in the factor-structure matrix, the number of factors to be sorted, the desired loading cutoff, and the name of the data files found in the virtual environment. These parameters, as opposed to the above data files, are entered interactively, facilitating repeated sorting of the same factor structure matrix with different sorting parameters.

Program Output. FACSORT output includes: (1) the percentage of common variance accounted for by each factor; (2) a separate table of variables loaded on multiple factors; (3) a listing of all variables not included in any of the sorted factor lists-these items possess nonsignificant factor loadings below the selected cutoff value; and (4) a listing of the variables with the significant factor loadings on each factor printed in descending order of loading magnitude with the associated variable descriptions.

Language and Computer. FACSORT is written in PASCAL 8000 with an accompanying operating exec. PASCAL 8000 is similar to other PASCAL versions (e.g., UCSD PASCAL), making it suitable for easy transfer to most microcomputers. The package is developed for use under the IBM VM/SP (CMS). Because FACSORT is designed as an interactive program, it requires that the user be familiar with basic CMS operations.

Documentation. The FACSORT User's Guide (Loh, Reynolds, Penzien, \& Tobin, 1985) provides a brief introduction to factor analysis interpretation, as well as detailed instructions for the use of the FACSORT package.

Availability. Copies of the FACSORT User's Guide and the FACSORT program and operating files (stored on magnetic tape or printed listing) can be obtained at cost from Russ Reynolds, Department of Psychology, Box 85, Porter Hall, Ohio University, Athens OH 45701. The user may supply his or her own magnetic tape.

\section{REFERENCE}

Loh, E. S., Reynolds, R. V. C., Penzien, D. B., Tobin, D. L. (1985). FACSORT(TM): Version 3.1, User's Guide. Unpublished manual, Ohio University.

(Revision accepted for publication April 17, 1986.) 